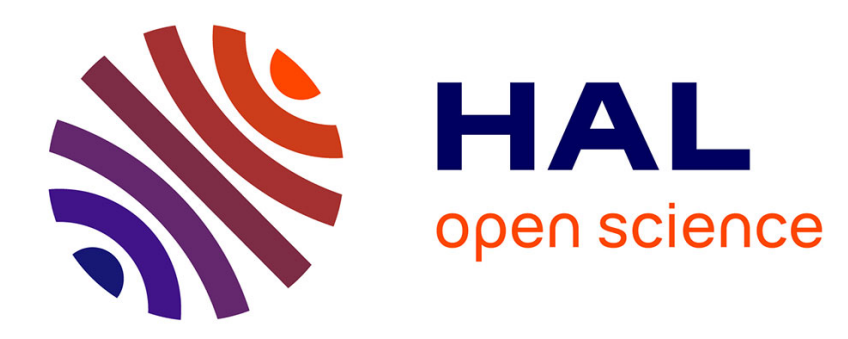

\title{
Variations sémantiques autour de la mer, d'Homère à Eschyle
}

Julie Sorba

\section{- To cite this version:}

Julie Sorba. Variations sémantiques autour de la mer, d'Homère à Eschyle. Gaïa - Revue interdisciplinaire sur la Grèce archaïque, 2015, 18, pp.311-323. 10.3406/gaia.2015.1666 . hal-01844317

\section{HAL Id: hal-01844317 https://hal.science/hal-01844317}

Submitted on 9 Aug 2018

HAL is a multi-disciplinary open access archive for the deposit and dissemination of scientific research documents, whether they are published or not. The documents may come from teaching and research institutions in France or abroad, or from public or private research centers.
L'archive ouverte pluridisciplinaire HAL, est destinée au dépôt et à la diffusion de documents scientifiques de niveau recherche, publiés ou non, émanant des établissements d'enseignement et de recherche français ou étrangers, des laboratoires publics ou privés. 
Variations sémantiques autour de la mer d'Homère à Eschyle

Julie Sorba, Université Grenoble Alpes - LiDiLEM

\section{Introduction}

La tragédie grecque se nourrit de l'épopée aussi bien dans le choix de ses motifs que dans celui de ses mots (Alaux 2006, p. 16-17). C'est pourquoi nous proposons de décrire dans les tragédies d'Eschyle l'évolution post-homérique d'unités lexicales. Nous nous intéresserons aux séquences phraséologiques incluant des lexies du champ de la 'mer'. Dans le corpus eschyléen,

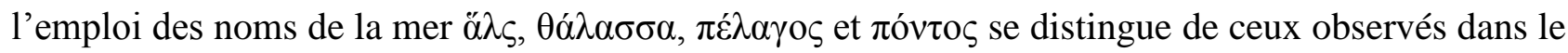
corpus homérique (voir Sorba 2008) ${ }^{1}$; nous avançons donc l'hypothèse que les lexies qui en sont issues présentent elles aussi des traits d'innovation.

\subsection{Cadre théorique et méthodologique}

La tragédie et l'épopée sont deux types de texte poétique en grande partie préconstruit dans leur schéma discursif et dans les expressions mobilisées. La phraséologie y joue un rôle clé car elle facilite, précisément par son caractère préconstruit, l'écriture. Les unités phraséologiques retenues dans le cadre de cette étude relèvent de la catégorie des collocations nominales ${ }^{2}$. La description que nous en proposons concerne les niveaux syntaxique et lexical (fonction et position spécifiques, associations privilégiées ${ }^{3}$ ), mais elle intègre également les propriétés énonciatives dans une approche du sens comme produit global de l'énoncé (voir Rastier 2011). Tous ces éléments

\footnotetext{
${ }^{1}$ Dans les tragédies d'Eschyle comme dans les épopées homériques, la lexie la plus fréquemment employée pour

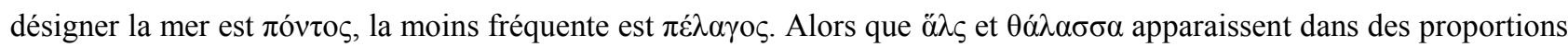
similaires chez Homère, l'emploi de $\theta \alpha \dot{\lambda} \alpha \alpha \sigma \sigma \alpha$ progresse nettement chez le tragique aux dépends de ö $\lambda$ s. L'étude

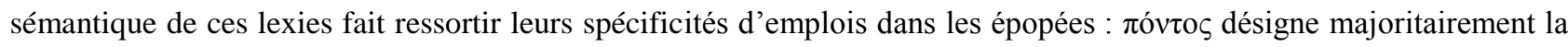

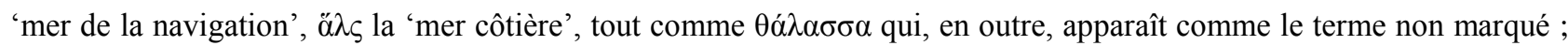
$\pi \hat{\varepsilon} \lambda \alpha \gamma o \varsigma$, enfin, est essentiellement utilisé pour désigner un espace lointain et dangereux, un lieu investi par les dieux (voir Sorba 2010). Le corpus eschyléen réorganise le matériau épique par le développement de nouvelles associations

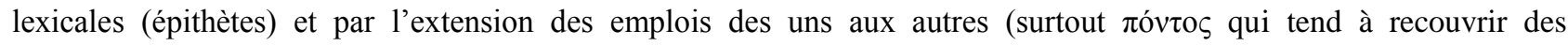
acceptions de $\alpha \ddot{\lambda} \varsigma$ et de $\theta \alpha \dot{\lambda} \lambda \alpha \sigma \sigma \alpha$, voir Sorba 2008).

${ }^{2}$ Une collocation est une cooccurrence de deux constituants linguistiques entretenant une relation sémantique et syntaxique privilégiée (voir Tutin et Grossmann 2003). Dans cette association, les deux lexies ont un statut inégal : une base autonome au plan sémantique est unie à un collocatif qui lui ajoute une caractérisation sans en modifier l'identité et qui voit son sens ainsi modifié.

${ }^{3}$ Ces données permettent d'élaborer le profil combinatoire de la lexie, c'est-à-dire la «structure schématique [de son] voisinage syntaxique et sémantique» (Blumenthal 2002, p. 115-116). Celui-ci comprend «l'ensemble des accompagnateurs stéréotypés du mot, porteurs d'associations typiques» (Blumenthal 2007, p. 19). Dans le cadre de la théorie du Lexical Priming, l'emploi d'un mot est lié à des associations sémantiques pré-activées (Hoey 2005, p. 13).
} 
permettent d'établir le profil discursif des lexies ${ }^{4}$ à partir duquel nous étudierons le traitement de la synonymie: la création de nouvelles lexies comble-t-elle un vide lexical ou bien celles-ci se substituent-elles à d'autres lexies dans le cadre d'emplois devenus similaires ? Notre objectif est donc double: (1) analyser le devenir des séquences épiques chez Eschyle et (2) tenter de comprendre ce qui a motivé l'emploi de nouvelles lexies dans les tragédies.

\subsection{Présentation du corpus}

Notre corpus est constitué des deux épopées homériques (Iliade, Odyssée) et des sept tragédies d'Eschyle conservées (Perses, Sept contre Thèbes, Suppliantes, Agamemnon, Choéphores, Euménides, Prométhée enchaîné $)^{5}$. Dans les deux cas, les textes mettent en œuvre une langue poétique versifiée et composite. Les données ont été extraites à l'aide du Thesaurus Linguae Graecae. Toutes les traductions fournies sont personnelles.

\subsection{Présentation des lexies}

Les unités phraséologiques sélectionnées relèvent du champ de la 'mer' et comportent toutes

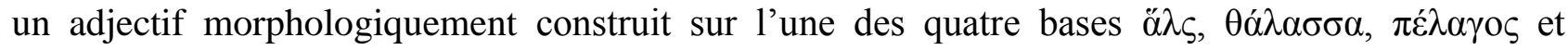

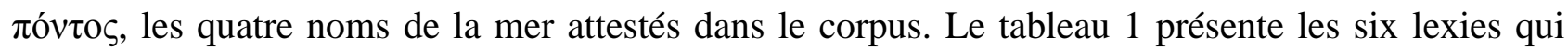
font l'objet de notre étude ${ }^{6}$ :

\begin{tabular}{|c|c|c|}
\hline & Homère $(\mathrm{H})$ & Eschyle (E) \\
\hline base $\ddot{\alpha} \lambda \varsigma$ & \multicolumn{2}{|c|}{ 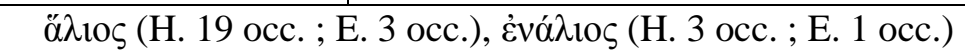 } \\
\hline base $\theta \alpha ́ \lambda \alpha \sigma \sigma \alpha$ & \multicolumn{2}{|c|}{$\theta \alpha \lambda \alpha ́ \sigma \sigma ı \varsigma$ (H. 2 осc. ; E. 3 осc.) } \\
\hline base $\pi \dot{\varepsilon} \lambda \alpha \gamma o \varsigma$ & & $\pi \varepsilon \lambda \alpha \dot{\alpha} \gamma 10 \varsigma$ (2 occ.) \\
\hline 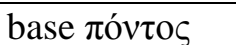 & & 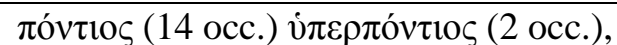 \\
\hline
\end{tabular}

Tableau 1 : Présentation des lexies de l'étude

\footnotetext{
${ }^{4}$ Sur la notion de profil discursif et sa modélisation fonctionnelle, voir Novakova et Sorba 2014.

${ }^{5}$ De datations trop diverses, les Hymnes ont été écartés du corpus, de même que les fragments des œuvres d'Eschyle, qui offraient un environnement textuel trop lacunaire pour appréhender le sens de la lexie.

${ }^{6}$ Nous avons écarté de l'étude les lexies non attestées chez Homère qui n'apparaissaient qu'une seule fois dans les

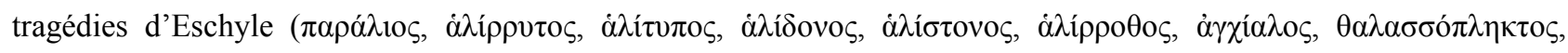

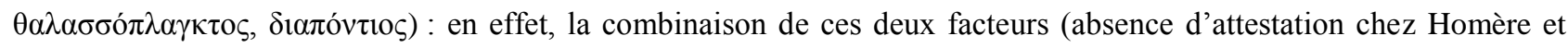
unique occurrence chez Eschyle) ne peut renseigner sur un phénomène récurrent.
} 
L’unité de cette série, composée de six formations hypostatiques nominales (voir Rousseau 2003), est assurée par la présence du suffixe $-10 \varsigma^{7}$. Dès lors, la variation sémantique permettant de discriminer des lexies traitées comme synonymes ${ }^{8}$ ne peut pas provenir du suffixe, mais bien de la base. Nous écartons également d'emblée le paramètre métrique car aucune des lexies n'est directement substituable à une autre. Enfin, la plus grande variété apparente des formations dans les tragédies où les quatre bases sont représentées doit être toutefois nuancée car l'absence de lexies chez Homère ne signifie pas que celles-ci n'étaient pas déjà en usage dans la langue ${ }^{9}$. L'objectif n'est donc pas tant de savoir si Eschyle a pratiqué la création lexicale que de décrire les nouveaux emplois que ses tragédies présentent par rapport au modèle homérique.

\section{Analyse des lexies}

Les six adjectifs apparaissent quasi exclusivement dans des séquences du type Nom (base) + Épithète (collocatif), ce qui est conforme à leur statut d'adjectifs relationnels. Nous analyserons d'abord le fonctionnement syntaxique de chacune des séquences qui comprennent les lexies de l'étude (profil syntaxique); puis nous étudierons les associations lexicales privilégiées à l'intérieur des collocations par les adjectifs (profil lexical) ; nous envisagerons enfin les paramètres énonciatifs de l'environnement textuel des lexies de l'étude (profil énonciatif).

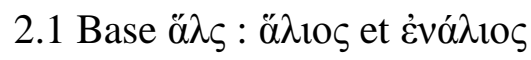

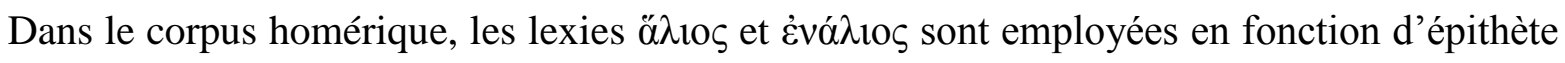
d'un nom animé (sauf 1 occ. voir infra). Le premier adjectif, ä $\lambda$ ıos (21 occ.), apparaît

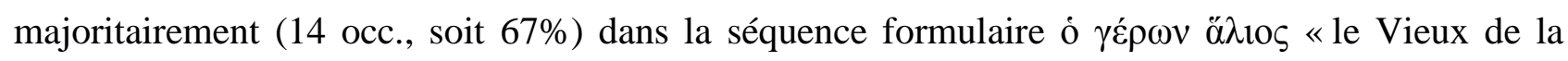
mer $»^{10}$. Quelques occurrences au féminin se rencontrent, d'une part, dans la qualification de ses

\footnotetext{
${ }^{7}$ La fonction essentielle de ce suffixe -10 est de former des adjectifs dérivés de noms ; sa très grande productivité en fait un morphème plutôt banal (Chantraine 1979, p. 37). On peut attribuer aux adjectifs en -10 la valeur sémantique très générale « qui est en relation avec, qui appartient à », mais « cette valeur est sans doute inhérente à la nature adjectivale de ces formes » (Rousseau 2003, p. 116-117).

${ }^{8}$ Le LfgrE postule une relation synonymique entre les lexies puisqu'il en fournit la même définition : «zum Meer gehörig » (s.v.)

${ }^{9}$ Par ex., le nom de personne mycénien po-ti-jo bâti sur le toponyme Пóvtıৎ (voir Duhoux et Morpurgo Davies 2011,

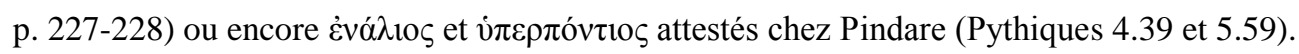

${ }^{10}$ Voir Iliade 1.538, 1.556, 18.141, 20.107, 24.562 ; Odyssée 4.349, 4.365, 4.384, 4.401, 4.542, 13.345, 24.58 (Nérée) ; Odyssée 17.140 (Protée) ; Odyssée 13.96 (Phorkys). Selon Perpillou (1973, p. 248), l'adjectif ö $\lambda$ ios n'est pas utilisé «pour ceux qui vivent de la mer ou travaillent sur la mer, car ils lui sont extérieurs, et loin d'en être issus, ils y vont : $\alpha \varkappa \lambda$ los. exprime une dépendance à l'égard de la mer, origine, possession, partie d'un tout ».
} 
filles, les Néréides $(6 \text { occ. })^{11}$, et, d'autre part, dans celle du nom du sable $(1 \text { occ. })^{12}$. Le second, Eivód d'animaux (3 occ., oiseaux et phoques) ${ }^{14}$. Les deux adjectifs apparaissent au sein de séquences phraséologiques spécifiques déterminant un référent animé, cantonné à des personnages divins bien

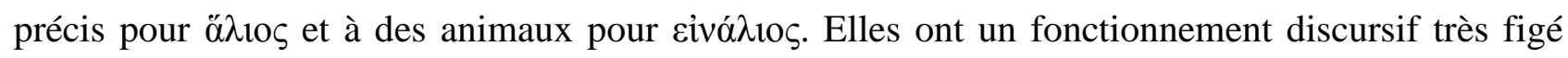

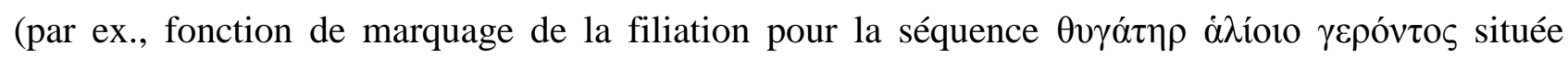
systématiquement après la coupe penthémimère). Dans la majorité des occurrences (76\%), les syntagmes appartiennent à la structure actancielle du verbe (actants syntaxiques sujet, complément direct ou indirect). Enfin, ces deux adjectifs dérivés revêtent l'acception de 'mer côtière' majoritairement actualisée par leur base ä $\lambda \varsigma$ dans l'épopée : c'est bien sur la zone littorale qu'on trouve sable, phoques et oiseaux et que Nérée et ses filles apparaissent aux hommes.

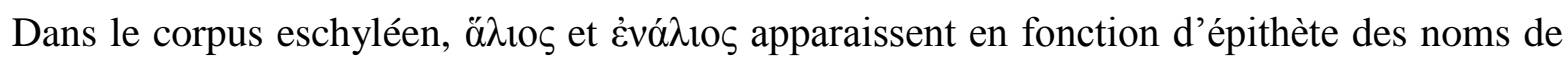

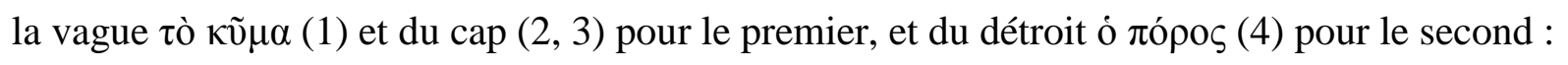

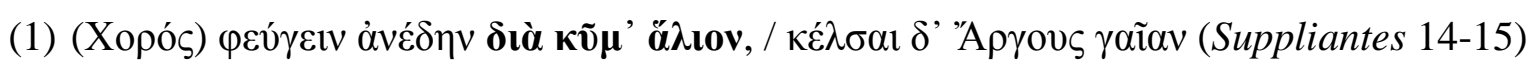

«Fuir de tout son être sur la vague marine et aborder sur la terre d'Argos. »

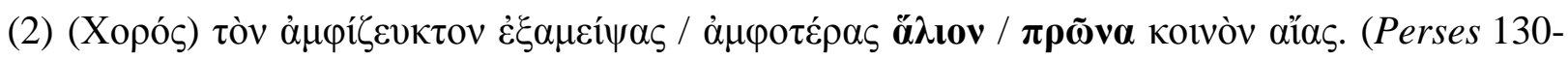
132 ) «[Xerxès] a franchi le cap marin en joignant les deux bords des deux continents. »

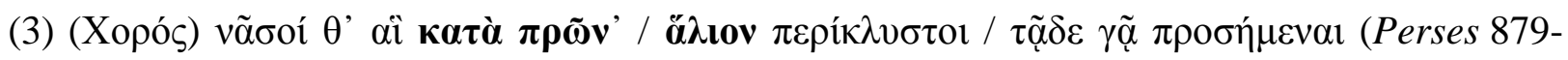
881) «Et les îles baignées par la mer, le long du cap marin, sont situées près de cette terre. »

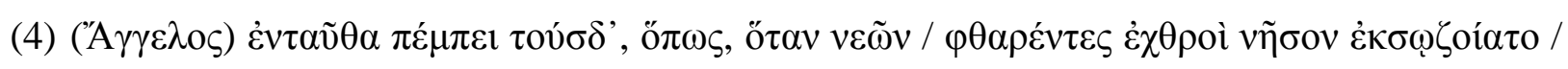

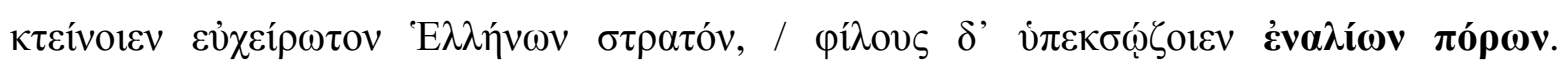
(Perses 450-453) « [Xerxès] les envoie là, afin que, si des ennemis naufragés se réfugiaient dans l'île, ils pussent massacrer l'armée des Grecs alors facile à soumettre tout en sauvant les leurs des détroits marins. »

Bien que ces séquences ne soient pas attestées sous cette forme dans le corpus homérique, elles peuvent néanmoins être rapprochées d'autres unités phraséologiques épiques dans lesquelles le génitif $\dot{\alpha} \lambda$ ó $\varsigma$ complète les noms du flot $\tau$ ò $\kappa \tilde{u} \mu \alpha$ et du détroit ó $\pi$ ó $\rho \circ \varsigma^{15}$. Dans ces cooccurrences, les

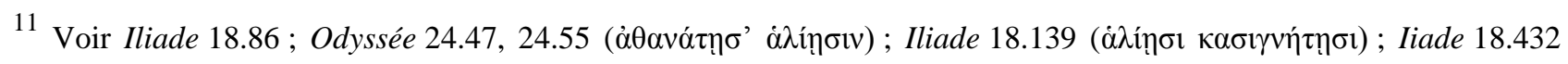

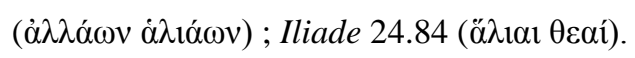

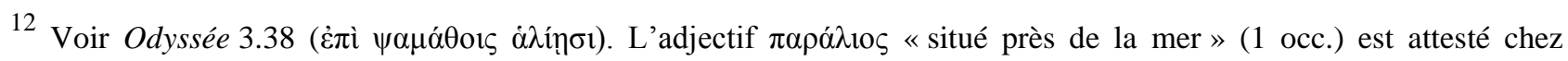

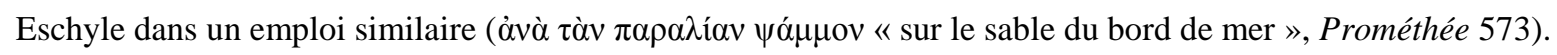

13 Voir Perpillou 2005, p. 275.

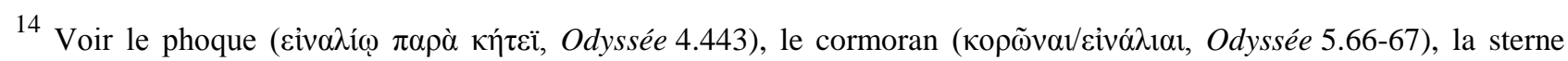

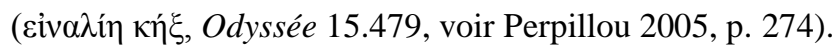

${ }^{15}$ Voir Iliade 6.136 ; Odyssée 12.68 ; 12.259 ; 22.387. Ces syntagmes ne sont pas attestés dans le corpus eschyléen.
} 


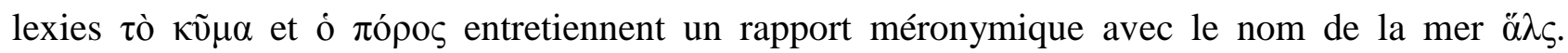
L'emploi de l'adjectif correspondant en (1) et en (4) relève alors d'une adaptation syntaxique et métrique de la séquence épique.

Néanmoins, les quatre séquences ainsi formées ne réfèrent plus à des personnages mais à des lieux au sein de récits mettant en scène la fuite des Danaïdes (1), le passage de la flotte perse (2) et sa défaite à Salamine (4) ou encore situant, dans un catalogue, différentes îles égéennes soumises par Xerxès (3). Celles-ci désignent ainsi une 'mer côtière', à l'exception de (1) qui évoque la mer de la navigation, autre emploi répertorié de ö $\lambda \varsigma$ dans l'épopée et dans la tragédie (Sorba 2010, p. $307-$ 311 ; Sorba 2008, p. 144). En outre, aucun profil syntaxique spécifique ne se détache dans le corpus eschyléen : dans les séquences (1) et (3), l'adjectif ö $\lambda$ io apparait dans un GP circonstant alors qu'en (2) et en (4), le syntagme contenant l'adjectif se présente comme un actant syntaxique du verbe. Sur le plan énonciatif, les séquences apparaissent dans les parties narratives chantées (1, 2, 3 ) ; elles ont majoritairement une fonction référentielle ${ }^{16}$, comme dans l'épopée.

\subsection{Base $\theta \alpha ́ \lambda \alpha \sigma \sigma \alpha: \theta \alpha \lambda \alpha ́ \sigma \sigma 10 \varsigma$}

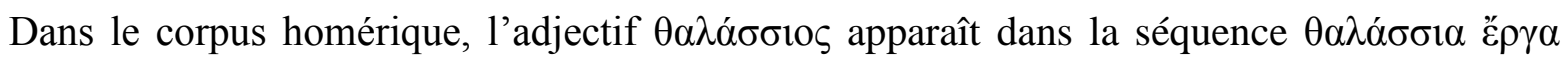
$(\mu \varepsilon \mu \eta \dot{\lambda} \lambda \varepsilon 1 / \mu \varepsilon \dot{\mu} \mu \eta \lambda \varepsilon v)$ «(s'occuper de)s travaux de la mer »(2 occ.), toujours placée après la coupe trochaïque, ce qui tend à en faire une unité phraséologique figée. Elle désigne, de manière périphrastique et métaphorique, l'art de la navigation, ignoré des Arcadiens (Iliade 2.614, description, catalogue des vaisseaux), et l'activité des cormorans (Odyssée 5.67, description de l'île de Calypso). L'adjectif conserve le sémantisme de la base $\theta \alpha \dot{\alpha} \lambda \alpha \sigma \sigma \alpha$. En tant que terme non marqué, elle peut en effet s'employer dans des contextes de navigation et de pêche animale. La séquence se présente comme une collocation à fonction référentielle.

Dans le corpus eschyléen, l'adjectif $\theta \alpha \lambda \alpha ́ \sigma \sigma 10 \varsigma$ est également employé comme épithète (6, 7), mais aussi de manière substantivée pour désigner les marins (5) :

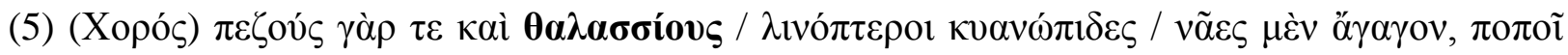
(Perses 558-560) «Fantassins et marins, les navires aux voiles de lin et à l'aspect sombre les ont emmenés, hélas !»

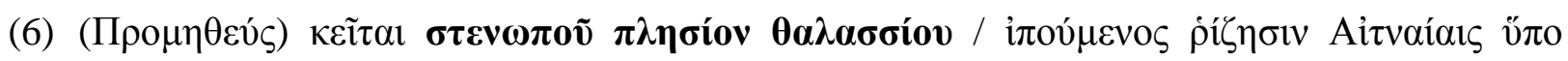

(Prométhée 364-365) «Il est étendu près d'un détroit marin, comprimé par les racines de l’Etna. »

\footnotetext{
${ }^{16}$ Les séquences phraséologiques à fonction référentielle se distinguent des séquences à fonction discursive qui servent à structurer l'organisation textuelle, des séquences à fonction interpersonnelle qui apparaissent dans un contexte dialogal ainsi que des routines sémantico-rhétoriques, énoncés stéréotypés renvoyant à des fonctions rhétoriques spécifiques (expliquer, persuader etc.). Pour une typologie des séquences phraséologiques, voir Tutin 2014, p. 30-34.
} 


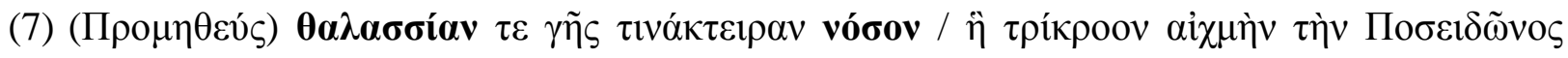

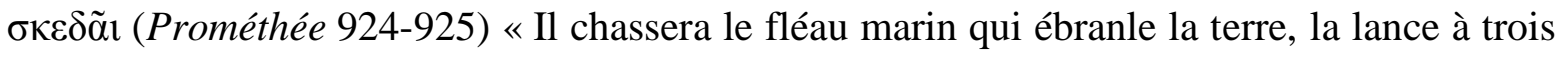
pointes de Poséidon. »

Employé seul, l'adjectif substantivé actualise le sens générique de la base $\theta \alpha ́ \lambda \alpha \sigma \sigma \alpha$ (5) grâce au système d'opposition dans lequel il entre (terre / mer). Comme épithète, il est associé de manière

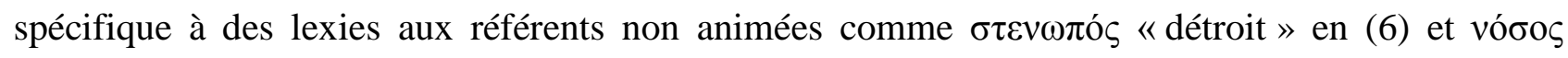
«fléau» en (7). Sur le plan syntaxique, la séquence appartient à la valence verbale $(5,7)$ ou s'intègre dans un GP circonstant $(6, \pi \lambda \eta \sigma i ́ o v)$. Enfin, $\theta \alpha \lambda \alpha ́ \sigma \sigma 10 \varsigma$ apparaît dans un environnement textuel narratif (expédition de Xerxès en (5), récit des malheurs de Typhée en (6), parties chantées) mais aussi au sein d'un discours de menace en (7), partie parlée). Ainsi, ces collocations référentielles peuvent participer $(5,6)$ ou non (7) à la progression narrative. Même si les emplois tragiques reprennent certaines propriétés des emplois épiques (noms à référent non animé, acceptions de la base), ils sont moins figés que ceux de l'épopée.

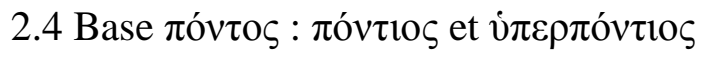

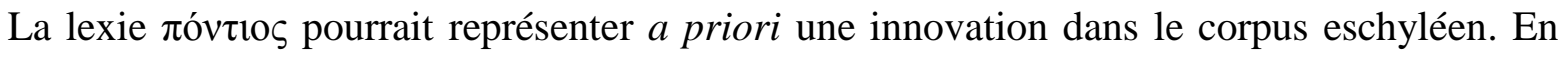
effet, elle n'est pas attestée dans le corpus épique homérique et Eschyle y recourt beaucoup plus fréquemment (14 occ.) qu'aux autres lexies de notre étude d'un sémantisme pourtant très proche. La

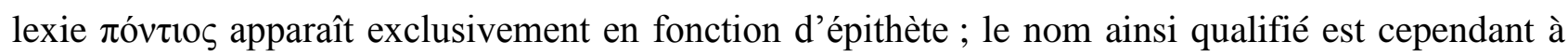
chaque fois différent, ce qui produit une séquence unique d'un point de vue morpho-syntaxique (voir tableau 2).

\begin{tabular}{|c|c|}
\hline & La séquence appartient à la structure actancielle \\
\hline nominatif (4 occ.) & 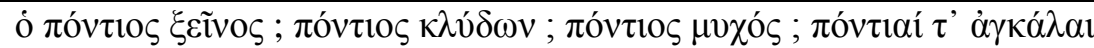 \\
\hline accusatif (2 occ.) & 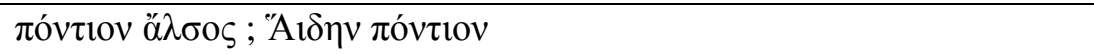 \\
\hline génitif (1 occ.) & 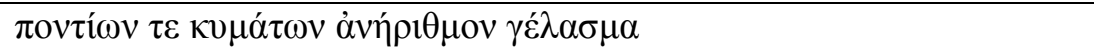 \\
\hline \multirow[t]{2}{*}{ datif (2 occ.) } & 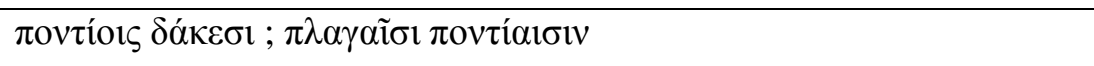 \\
\hline & La séquence est dans un circonstant (GP ou non) \\
\hline génitif GP (2 occ.) & 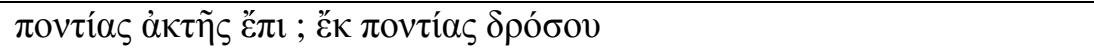 \\
\hline datif seul (2 occ.) & 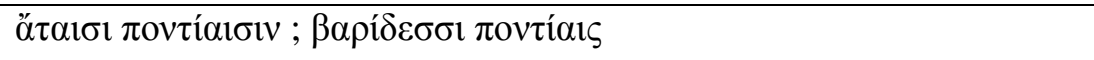 \\
\hline datif GP (1 occ.) & 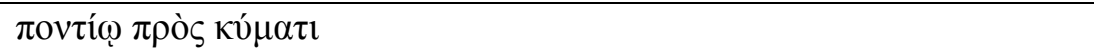 \\
\hline
\end{tabular}

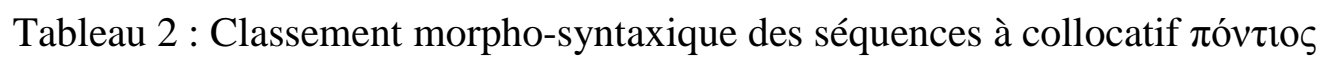

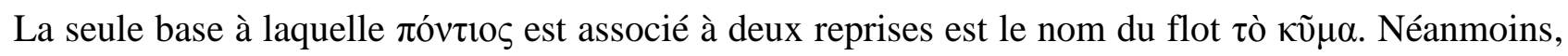
la séquence ainsi formée apparait tantôt dans un GP en fonction de circonstant (8), tantôt dans un GN en fonction de complément du nom (9) : 


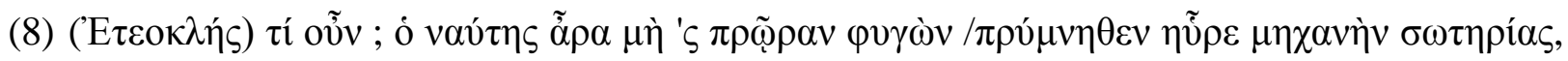

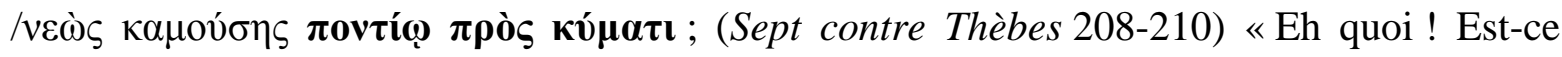
donc en fuyant de la proue à la poupe que le marin trouve une stratégie de salut, alors que le bateau souffre face à la vague marine ? »

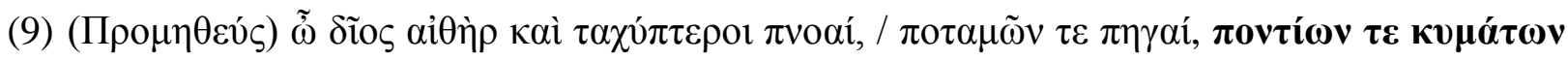

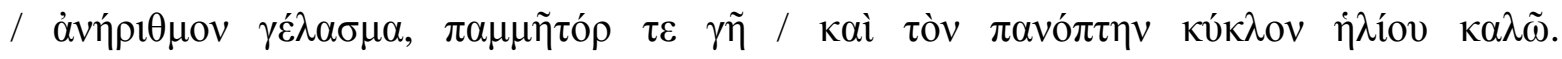
(Prométhée 88-90) «Ô Éther divin et vents aux ailes rapides, sources des fleuves et sourire innombrable des vagues marines, terre mère de tous les êtres et cercle du soleil qui voit tout, je vous invoque!»

À première vue, la lexie entre difficilement dans le cadre d'une séquence phraséologique. Néanmoins, le caractère très codifié du morceau textuel dans lequel la séquence apparait - énoncé de type gnomique (8) et invocation des éléments (9) - pourrait constituer un indice de sa nature phraséologique.

Par ailleurs, malgré l'hétérogénéité des constructions syntaxiques des séquences à collocatif $\pi$ óvtıs, celui-ci s'associe exclusivement à une base dont le référent est non humain (inanimé ou êtres merveilleux). Ces séquences peuvent être classées selon un critère lexical permettant de déterminer les champs sémantiques des associations privilégiées. Un premier groupe rassemble les

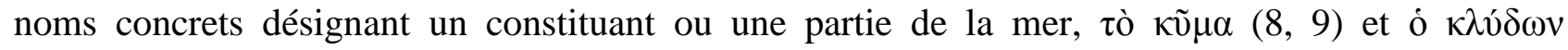

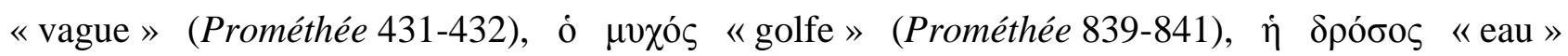

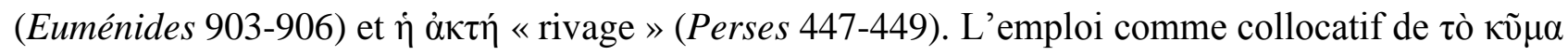
- emploi commun avec ö $\lambda \varsigma$, voir (1) - repose sur un rapport méronymique entre un élément et ses parties. C'est un emploi non attesté par les données homériques qui ne présentent jamais une

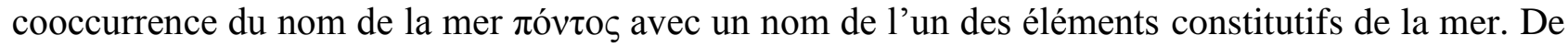

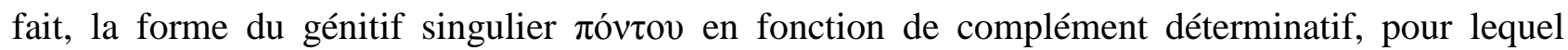

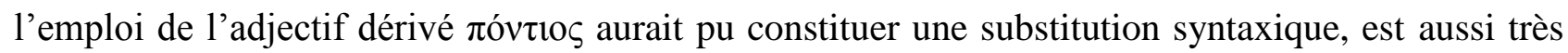
rare dans les épopées ${ }^{17}$. Quant aux séquences épiques désignant le rivage de la mer, elles utilisent

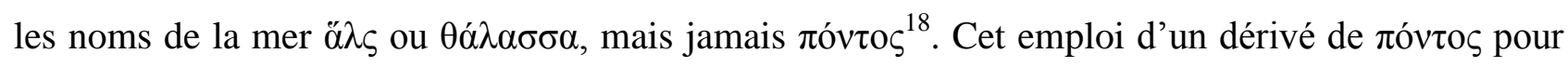
qualifier la zone du littoral est donc une innovation par rapport au corpus homérique ${ }^{19}$.

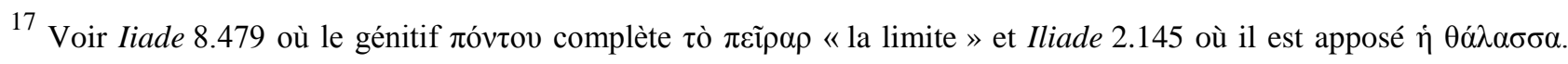
Pour une explication de ces faits voir Sorba 2010, p. 424.

${ }^{18}$ Voir par ex., les séquences homériques désignant le rivage de la mer qui associent les noms de la mer ő $\lambda \varsigma$ et $\theta \alpha ́ \lambda \alpha \sigma \sigma \alpha$

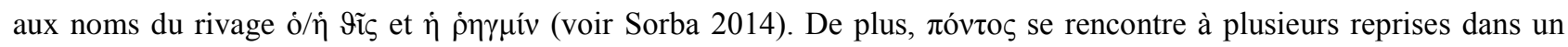
contexte actualisant l'acception 'haute mer' et l'opposant à une autre lexie désignant la zone côtière (voir, par ex., Iliade 1.349-350 et 23.125).

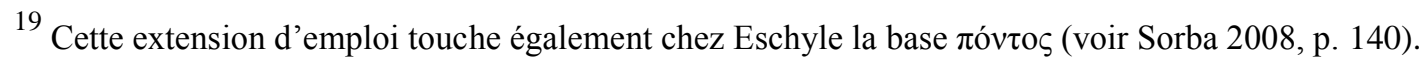




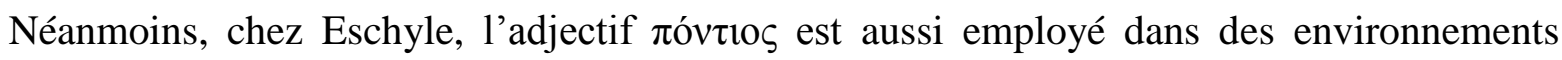

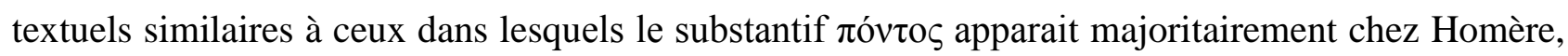
à savoir des contextes où la mer est présentée comme le lieu de la navigation et des dangers ${ }^{20}$. La

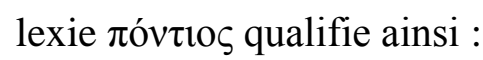

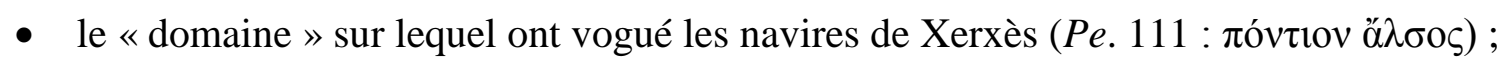

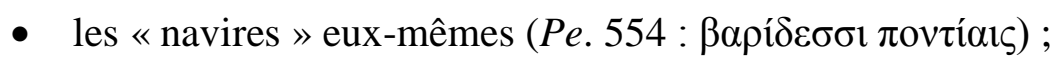

- les «coups » reçus par la flotte grecque (Pe.906: $\pi \lambda \alpha \gamma \alpha i ̃ \sigma ı ~ \pi o v \tau i ́ \alpha ı \iota v)$ et les «malheurs »

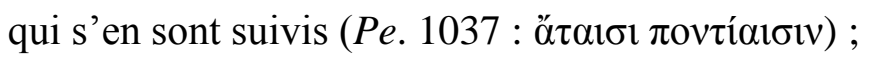

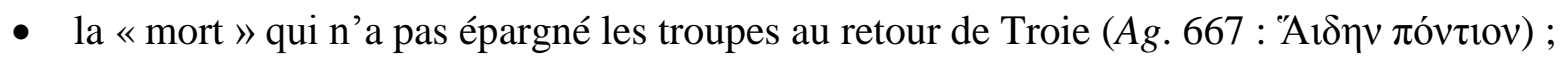

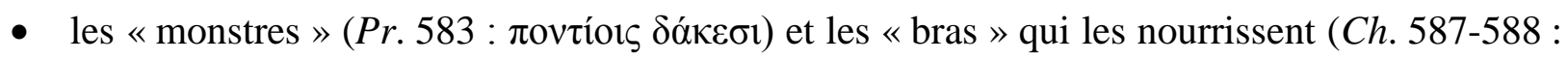

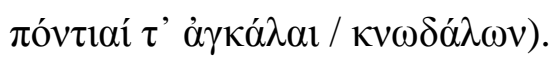

Dans les épopées homériques, ces associations lexicales ne sont pas attestées, mais l'environnement

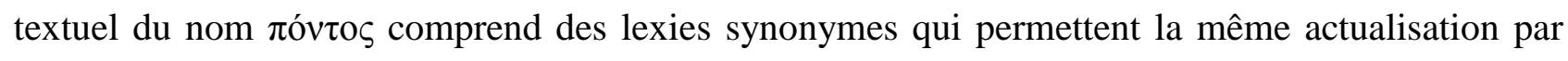

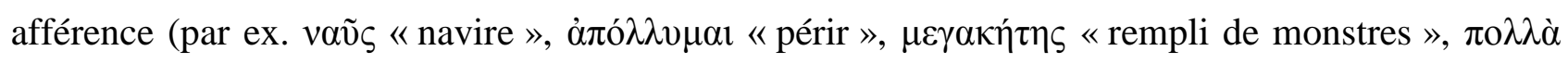

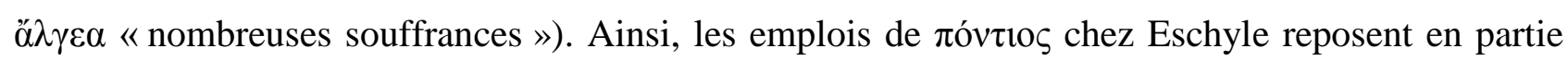

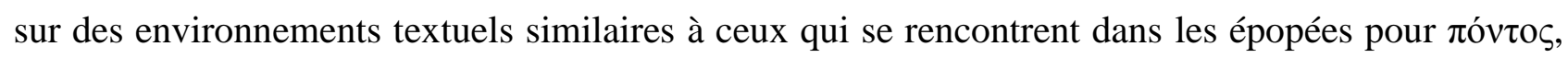
la base dont il est dérivé.

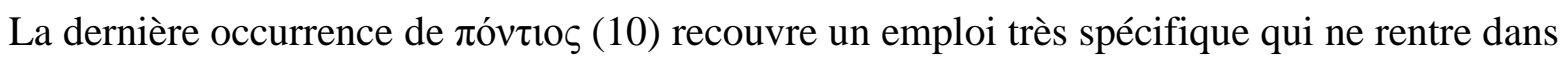
aucune des deux catégories proposées ci-dessus pour le classement des associations lexicales privilégiées :

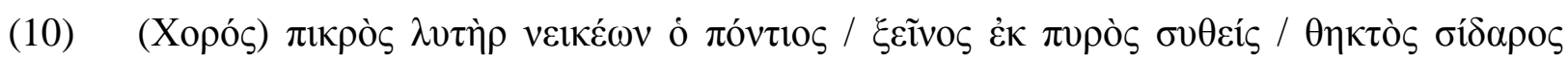
(Sept contre Thèbes 941-943) «Cruel arbitre de leurs querelles [fut] l'étranger venu de la mer, le fer qui sort aiguisé du feu. »

La lexie est utilisée pour exprimer la provenance du métal qui a servi à forger les épées avec lesquelles les deux frères se sont entretués. Elle revêtirait alors une valeur toponymique pour désigner le Pont-Euxin et ses gisements de fer très réputés dans 1'Antiquité ${ }^{21}$. Cette interprétation

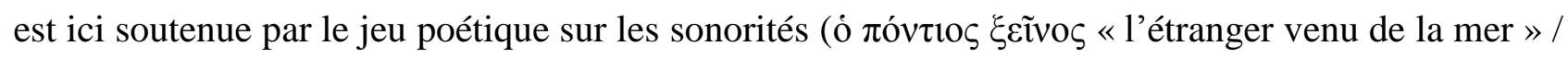

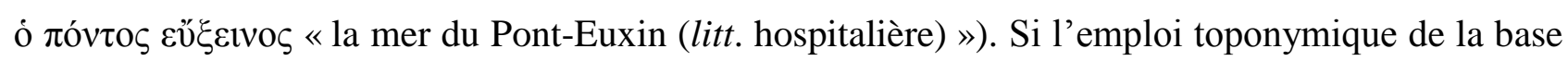

\footnotetext{
${ }^{20}$ Dans les épopées homériques, les sèmes afférents /navigation/ et /danger/ sont certes actualisés dans le sémème des

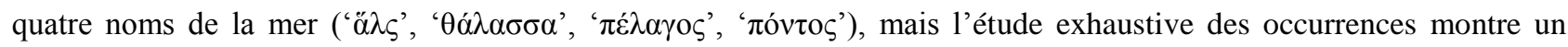

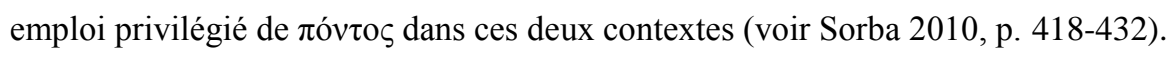

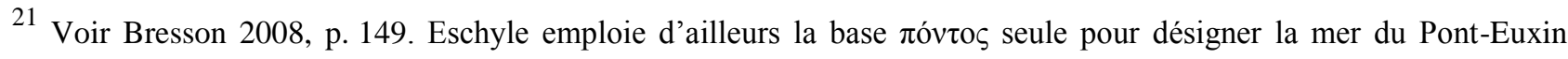

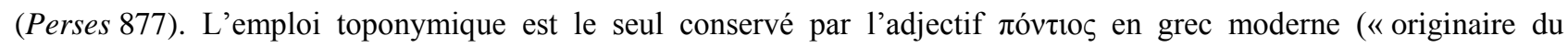
Pont »).
} 
$\pi$ óvtos n'est pas inconnu dans les épopées ${ }^{22}$, elle est seulement attestée dans le composé

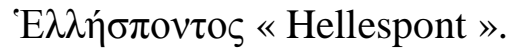

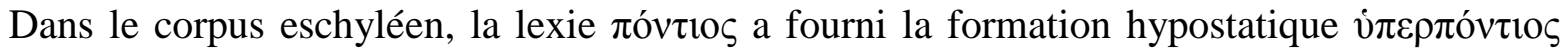

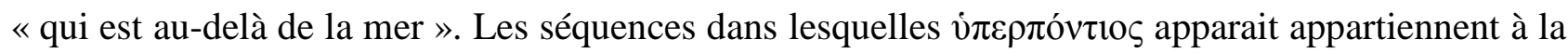
valence du verbe (groupe sujet en (12) et groupe COD en (11)) :

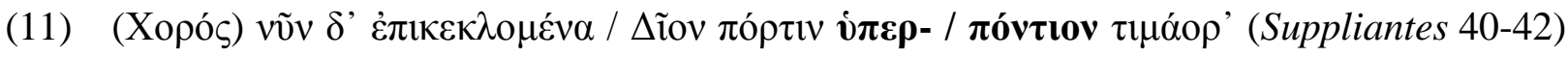
«Et maintenant après avoir appelé le jeune veau issu de Zeus, mon défenseur qui est au-delà de la mer...»

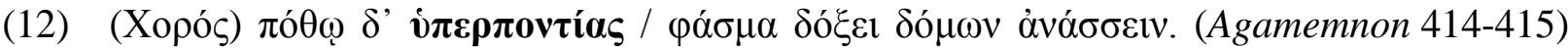

«Et à cause du désir, le fantôme de celle qui est [désormais] au-delà de la mer semblera régner sur ce palais. »

Le collocatif í $\pi \varepsilon \rho \pi o ́ v \tau t o \varsigma$ est associé des bases nominales ayant pour référent un être humain,

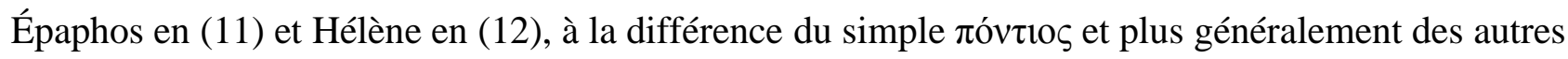

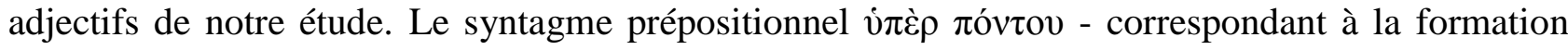

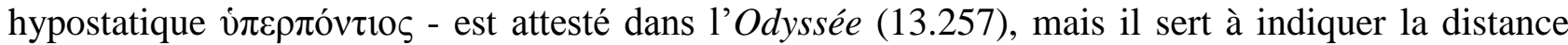

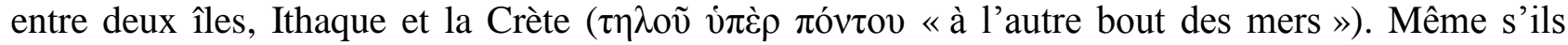
semblent se correspondre, syntagme prépositionnel (SP) et forme hypostatique relèvent de deux domaines différents, celui de la syntaxe et celui du lexique : le SP exprime la circonstance d'un procès quand l'adjectif construit sur ce SP exprime une qualité ou une essence ${ }^{23}$. Ainsi, Eschyle combine les deux éléments déjà utilisés par Homère, mais sous une forme et dans un emploi

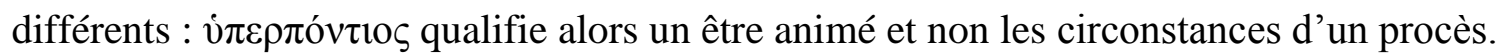

\subsection{Base $\pi \dot{\lambda} \lambda \alpha \gamma o \varsigma: \pi \varepsilon \lambda \alpha ́ \gamma 10 \varsigma$}

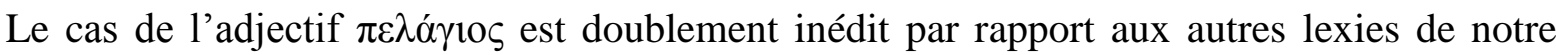
étude. En effet, il n'est pas attesté dans le corpus homérique pour une raison métrique car il est impossible à utiliser dans un hexamètre dactylique ; en outre, c'est le seul à servir d'épithète à un

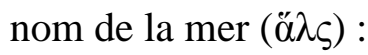

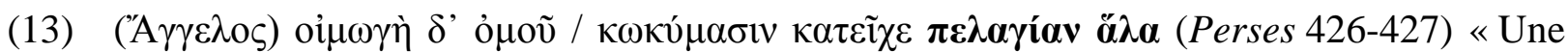
plainte mêlée de gémissements envahissait l'étendue marine. »

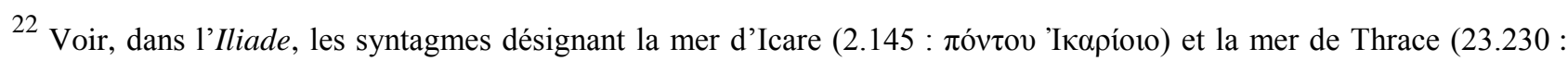

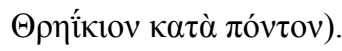

${ }^{23}$ Voir à ce propos Rousseau 2003, p. 170.
} 


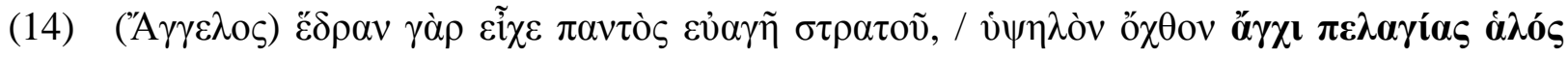
(Perses 466-467) «Il se tenait en une place dégagée offrant une vue sur toute l'armée, une hauteur escarpée près de l'étendue marine. »

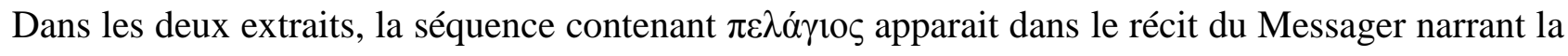
déroute de la flotte perse à Salamine : elle assure ainsi une fonction référentielle dans la progression narrative. Syntaxiquement, les deux occurrences ne présentent pas le même profil : en (13), la séquence appartient à la structure actancielle du verbe (accusatif complément), tandis qu'en (14), elle se trouve dans un GP au génitif. Cette séquence composée de deux lexies a priori sémantiquement redondantes s'inscrit dans un environnement textuel déployant ce procédé : dans le même vers en (13), se produit une répétition lexicale à valeur intensive oỉ $\mu \gamma \gamma \grave{\text { à } \kappa \omega ́ \kappa v \mu \alpha ~}$ «gémissement, lamentation »; on retrouve le même phénomène en (14) avec vं $\psi \eta \lambda$ òv/ő $\chi \theta o v$ « haut/hauteur ». Enfin, la séquence d'Eschyle est moins inédite qu'elle n'y parait car elle se

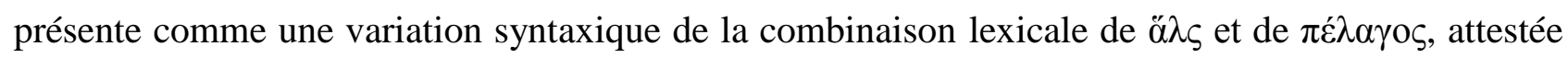
dans le syntagme homérique $\dot{\alpha} \lambda \grave{s} \varsigma \dot{\varepsilon} v \pi \varepsilon \lambda \dot{\alpha} \gamma \varepsilon \sigma \sigma l$ « sur l'étendue de la mer $»^{24}$.

\section{Conclusion}

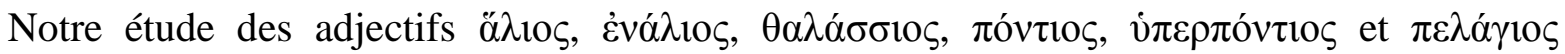
montre que le matériau épique a été largement réinvesti dans les tragédies d'Eschyle. Cependant, aucun emploi direct n'est attesté puisque les séquences ne dupliquent pas littéralement un modèle épique. En revanche, Eschyle propose une variation syntaxique des modèles relevés chez Homère :

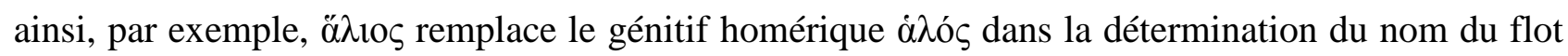

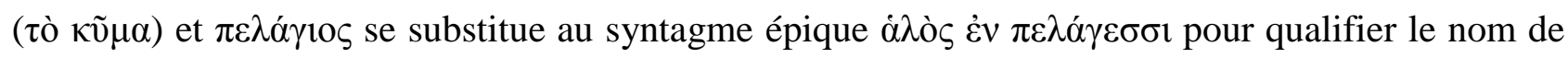

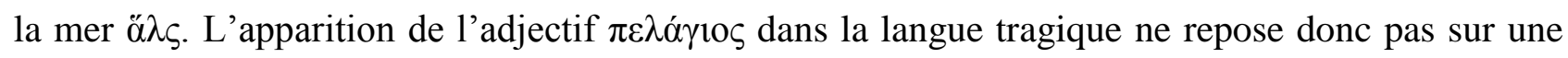
innovation sémantique (sa base $\pi \varepsilon ́ \lambda \alpha \gamma o \varsigma$ est attestée chez Homère dans la même association lexicale avec $\ddot{\alpha} \lambda \varsigma$ ), mais seulement sur la possibilité métrique de le faire entrer dans le vers tragique (ce que le schéma métrique du vers épique lui interdisait).

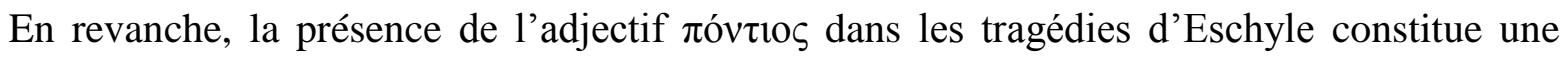
innovation par rapport au matériau épique. La lexie peut certes actualiser les mêmes traits

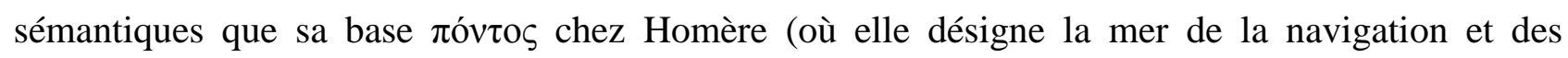
dangers), mais elle a élargi son sens en adoptant l'acception de 'mer côtière', usuellement actualisée par les noms de la mer ő $\lambda \varsigma$ et $\theta \alpha \dot{\lambda} \alpha \sigma \sigma \alpha$ (et par leurs dérivés). De fait, l'élargissement du sens de

\footnotetext{
${ }^{24}$ Voir Odyssée 5.335 (récit de la théophanie d'Ino-Leucothée). Notre traduction tente de rendre compte de l'idée d'immensité spatiale actualisée dans les emplois homériques de $\pi \varepsilon ́ \lambda \alpha \gamma o \varsigma$ (Sorba 2010, p. 401-410). Nous conservons cette traduction «étendue marine» pour les séquences eschyléennes qui prennent racine dans la phraséologie homérique.
} 


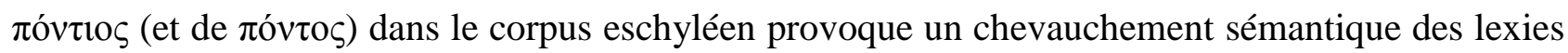

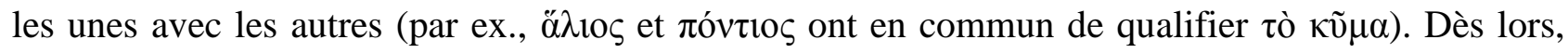
comment distinguer les lexies sémantiquement très proches les unes des autres ?

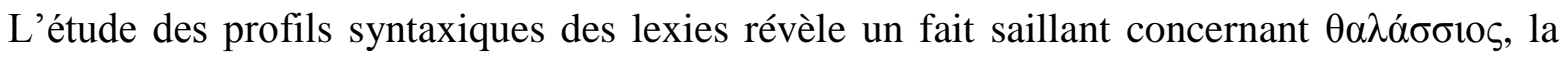
seule utilisée pour désigner des êtres humains, en l'occurrence les marins. Tous les autres adjectifs renvoient à des référents non animés ou non humains. L'analyse des profils lexicaux indiquent

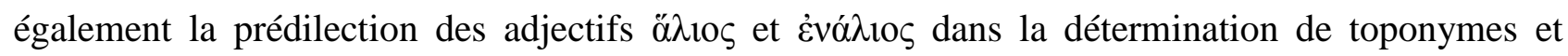
l'emploi unique de $\pi \varepsilon \lambda \alpha ́ \gamma 10 \varsigma$ comme collocatif de la base ő $\lambda$ s. Aucun élément spécifique n'a pu être dégagé des profils énonciatifs : toutes les collocations dans lesquelles se rencontrent les lexies de notre étude assurent une fonction référentielle au service de la progression narrative (la majorité d'entre elles (60\%) apparaissent d'ailleurs dans les parties parlées).

Par conséquent, dans les tragédies, les lexies du champ de la 'mer' maintiennent certaines acceptions spécifiques qui permettent encore de les distinguer les unes des autres. Enfin, la reprise systématique avec variation, fût-elle infime, des modèles attestés chez Homère est une manière pour le poète de mettre à la fois à distance et en miroir la langue tragique avec la langue épique.

Bibliographie

AlauX Jean, Lectures tragiques d'Homère, Paris, Belin, 2006.

Blumenthal Peter, «Profil combinatoire des noms. Synonymie distinctive et analyse contrastive », Zeitschrift für französische Sprache und Literatur, 112, 2, 2002, p. 115-138.

Blumenthal Peter, «Sciences de l'homme vs sciences exactes : combinatoire des mots dans la vulgarisation scientifique », Revиe française de linguistique appliquée, 12, 2, 2007, p. 1528.

Bresson Alain, L'économie de la Grèce des cités (fin VI $I^{e} I^{e r}$ siècle a. C.), Paris, A. Colin, 2008.

Chantraine Pierre, La formation des noms en grec ancien, Paris, Klincksieck, 1979.

Duhoux Yves et Morpurgo Davies Anna (dir.), A Companion to Linear B: Mycenaean Greek Texts and their World, vol. 2, Louvain-la-Neuve, Peeters, 2011.

HoEY Michael, Lexical Priming. A New theory of Words and Language, Londres-New York, Routledge, 2005.

NOVAKOVA Iva et SORBA Julie, «L'émotion dans le discours. À la recherche du profil discursif de stupeur et de jalousie », dans P. Blumenthal, I. Novakova, D. Siepmann (éd.), Les émotions dans le discours. Emotions in discourse, Francfort sur le Main, Peter Lang, 2014, p. 161-173.

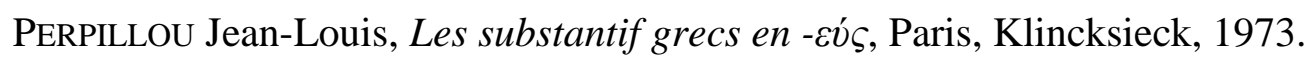

PerPILlou Jean-Louis, « Hypostases homériques », Revue de Philologie, 79, 2, 2005, p. 267-277. 
RASTIER François, La mesure et le grain. Sémantique de corpus, Paris, H. Champion, 2011.

ROUSSEAU Nathalie, Les formations hypostatiques nominales à premier élément prépositionnel en grec ancien, de l'époque archaïque à la fin de l'époque classique, Thèse de doctorat dactylographiée, Paris IV, 2003.

Lexikon des frühgriechischen Epos (LfgrE), B. Snell (éd.), Göttingen, Vandenhoeck \& Ruprecht, 1979-.

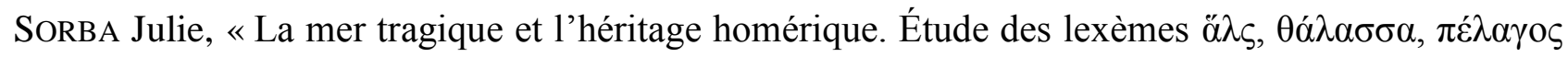

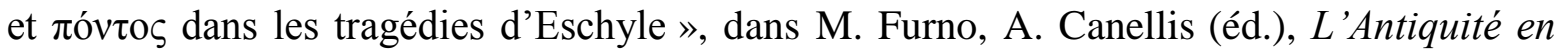
ses confins. Mélanges en l'honneur de Benoît Gain, ELLUG, Grenoble, 2008, p. 139-149.

SORBA Julie, Le vocabulaire de la mer en latin, grec ancien et indo-aryen ancien, Thèse de doctorat dactylographiée, École Pratique des Hautes Études, IV section: Sciences historique et philologique, Paris, 2010.

SORBA Julie, «Le rivage de la mer ou la ligne des brisants ? Vie et mort du lexème grec dans D. Ablali, S. Badir, D. Ducard (dir.), Documents, textes, auvres : perspectives sémiotiques, Rennes, PUR, 2014, p. 249-261.

Thesaurus Linguae Graecae Canon of Greek Authors and Works, L. Berkowitz et K.A. Squitier (éd.), New York/Oxford, OUP, 1990, 3 éd.

TUTIN Agnès, «La phraséologie transdisciplinaire des écrits scientifiques : des collocations aux routines sémantico-rhétoriques », dans A. Tutin, F. Grossmann (éd.), L'écrit scientifique : du lexique au discours. Autour de Scientext, Rennes, PUR, 2014, p. 27-44.

Tutin Agnès et Grossmann Francis, Les collocations. Analyse et traitement, Amsterdam, De Werelt, 2003.

Résumé / abstract

L'étude propose de décrire, dans les tragédies d'Eschyle, les séquences phraséologiques, héritées ou non du corpus épique, incluant des lexies du champ de la 'mer' (les adjectifs ä $\lambda$ ıos,

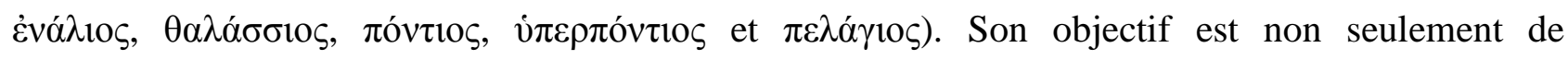
comprendre les évolutions de ces unités, mais aussi d'appréhender le traitement de la synonymie dans ce champ. À l'interface de la syntaxe et de la sémantique, l'analyse des lexies intègre également la description de leurs propriétés discursives dans une approche du sens comme un produit global de l'énoncé. Enfin, elle permet d'apprécier la virtuosité poétique d'Eschyle qui remodèle sans cesse le matériau épique.

In this study of lexical semantics in the Aeschylus' tragedies, we describe the phraseologic units which include some words referring to the 'sea' (especially the adjectives ö $\lambda$ ıos, غ่vó $\lambda$ ıo

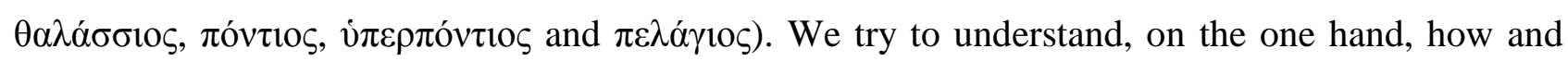


why the sequences used by Homer change and, on the other hand, how and why another units, not found in the epic corpora, are used in Aeschylus' tragedies. Afterwards, we propose to explain how work the synonymous links between these words. Thus, we approach the meaning as a global product of the utterance, analyzing the syntactic, lexical and enunciative levels. Finally, the poetic brilliance of Aeschylus, who reinvent consistently homeric legacy, can be observed through this study.

Mots-clés / key-words (From Homer to Aeschylus : lexical variations about the sea) sémantique lexicale, mer, Eschyle, Homère, ö $\lambda \iota^{\circ} \varsigma, \theta \alpha \lambda \alpha ́ \sigma \sigma 10 \varsigma, \pi o ́ v \tau 10 \varsigma, \pi \varepsilon \lambda \alpha ́ \gamma 10 \varsigma$.

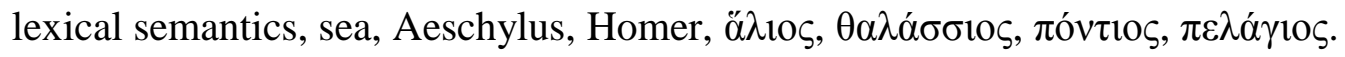

